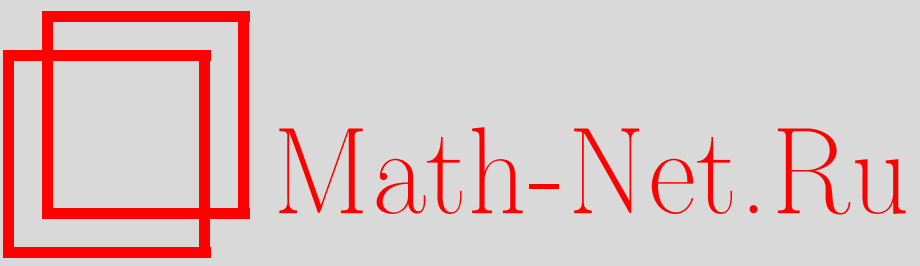

B.

И.

Денисов,

И. П. Денисова, С. И. Свертилов, Нелинейно-электродинамическое запаздывание электромагнитных сигналов, распространяющихся в плоскости магнитного меридиана пульсаров и магнетаров, ТМФ, 2004, том 140, номер 1, 128-138

DOI: https://doi.org/10.4213/tmf76

Использование Общероссийского математического портала Math-Net.Ru подразумевает, что вы прочитали и согласны с пользовательским соглашением

http://www.mathnet.ru/rus/agreement

Параметры загрузки:

IP : 3.81 .55 .215

26 апреля 2023 г., 09:20:53 
ТЕОРЕТИЧЕСКАЯ

И МАТЕМАТИЧЕСКАЯ

ФИЗИКА

Том 140, № 1

июль, 2004

(С) 2004 г. В.И. Денисов* , И.П. Денисова ${ }^{\dagger}$, С. И. Свертилов*

\section{НЕЛИНЕЙНО-ЭЛЕКТРОДИНАМИЧЕСКОЕ ЗАПАЗДЫВАНИЕ ЭЛЕКТРОМАГНИТНЫХ СИГНАЛОВ, РАСПРОСТРАНЯЮШИХСЯ В ПЛОСКОСТИ МАГНИТНОГО МЕРИДИАНА ПУЛЬСАРОВ И МАГНЕТАРОВ}

В параметризованной постмаксвелловской электродинамике вакуума проведен анализ распространения электромагнитных волн в плоскостях магнитных меридианов нейтронных звезд, обладающих сильным магнитным полем. Источником этих электромагнитных волн является изгибное рентгеновское и гамма-излучение высокоэнергетичных электронов, происходящее в областях магнитных полюсов нейтронной звезды. Показано, что в случае непрерывного излучения запаздывание медленной нормальной моды электромагнитных волн по сравнению с быстрой модой приводит к тому, что временна́я зависимость принимаемого излучения одной поляризации будет сдвинута относительно временно́й зависимости интенсивности рентгеновского и гамма-излучения ортогональной ей поляризации. В случае излучения отдельных рентгеновских и гамма-импульсов эффект запаздывания приведет к тому, что принимаемые импульсы будут иметь переменную по длине поляризацию, причем передняя часть всех импульсов будет поляризована перпендикулярно плоскости магнитного экватора нейтронной звезды. Отмечено, что современный уровень развития экспериментальной техники позволяет, в принципе, наблюдать проявления эффекта запаздывания сигналов, имеющих различную поляризацию.

Ключевые слова: нелинейная электродинамика вакуума, нейтронная звезда, пульсар, магнетар, постмаксвелловские параметры, двулучепреломление, нелинейно-электродинамическое запаздывание сигнала.

\section{1. ВВЕДЕНИЕ}

Лазерные эксперименты [1], проведенные на электронном ускорителе в Стэнфорде, показали, что электродинамика вакуума является нелинейной теорией. Уравнения

\footnotetext{
* Московский государственный университет, Москва, Россия. E-mail: denisov@srd.sinp.msu.ru; sis@coronas.ru

${ }^{\dagger}$ Российский государственный технологический университет, Москва, Россия
} 
электромагнитного поля в нелинейной электродинамике вакуума совпадают с нелинейными уравнениями электродинамики сплошных сред

$$
\begin{array}{rlrl}
\operatorname{rot} \mathbf{H} & =\frac{1}{c} \frac{\partial \mathbf{D}}{\partial t}, & \operatorname{div} \mathbf{D}=0, \\
\operatorname{rot} \mathbf{E}=-\frac{1}{c} \frac{\partial \mathbf{B}}{\partial t}, & \operatorname{div} \mathbf{B}=0,
\end{array}
$$

где явный вид материальных уравнений $\mathbf{D}=\mathbf{D}(\mathbf{B}, \mathbf{E})$ и $\mathbf{H}=\mathbf{H}(\mathbf{B}, \mathbf{E})$ зависит от выбора лагранжиана,

$$
\mathbf{D}=4 \pi \frac{\partial L}{\partial \mathbf{E}}, \quad \mathbf{H}=-4 \pi \frac{\partial L}{\partial \mathbf{B}} .
$$

В настояшее время в научной литературе рассматривается несколько различных моделей нелинейной электродинамики вакуума (1). В приближении слабого поля в рамках этих моделей лагранжиан в псевдоевклидовом пространстве-времени может быть записан в параметрическом виде [2] как

$$
L=\frac{1}{8 \pi}\left\{\left[\mathbf{E}^{2}-\mathbf{B}^{2}\right]+\xi\left[\eta_{1}\left(\mathbf{E}^{2}-\mathbf{B}^{2}\right)^{2}+4 \eta_{2}(\mathbf{B E})^{2}\right]\right\}+O\left(\eta \xi^{2} \mathbf{B}^{6}\right)-\rho \varphi+\frac{1}{c}(\mathbf{j} \mathbf{A}),
$$

где $\xi=1 / B_{q}^{2}, B_{q}=m^{2} c^{3} /(e \hbar)=4.41 \cdot 10^{13} \Gamma c$ - характерное квантово-электродинамическое значение, а величина безразмерных постмаксвелловских параметров $\eta_{1}$ и $\eta_{2}$ зависит от выбора модели.

В частности, в нелинейной электродинамике Гейзенберга-Эйлера параметры $\eta_{1}$ и $\eta_{2}$ имеют вполне конкретные значения, $\eta_{1}=\alpha /(45 \pi)=5.1 \cdot 10^{-5}, \eta_{2}=7 \alpha /(180 \pi)=$ $9.0 \cdot 10^{-5}$, в то время как в теории Борна-Инфельда они выражаются формулой $\eta_{1}=$ $\eta_{2}=a^{2} B_{q}^{2} / 4$, где для постоянной $a^{2}$ известна лиш оценка снизу: $a^{2}>1.2 \cdot 10^{-32} \Gamma^{-2}$.

Наиболее ярко эффекты нелинейной электродинамики вакуума должны проявляться в сильных электромагнитных полях, характерных для пульсаров и магнетаров. Эти нейтронные звезды, кроме того, являются интенсивными источниками рентгеновского и гамма-излучения.

Одним из основных механизмов генерации рентгеновского и гамма-излучения быстро вращающимися пульсарами и магнетарами является изгибное излучение, вид синхротронного излучения высокоэнергетичных электронов, движущихся вдоль магнитных силовых линий [3]. Ускорение электронов обеспечивается компонентами электрического поля, параллельными магнитному полю В. Такие области, в которых $\mathbf{E B} \neq 0$, могут существовать, в частности, вблизи поверхности нейтронной звезды над полярными шапками [4], а также вдоль так называемой поверхности "нулевого заряда" ( $\mathbf{\Omega}=0$, где $\boldsymbol{\Omega}$ - вектор угловой скорости врашения нейтронной звезды), которая разделяет замкнутые и разомкнутые силовые линии магнитного поля [5]. Предполагается, что в случае молодых пульсаров с сильным ( $B \geqslant 10^{13} \Gamma$ c) магнитным полем высокоэнергетичные электроны и связанное с ними жесткое излучение образуются в узком слое, находящемся вблизи магнитных полюсов на расстояниях, не превышаюших 0.5-1 радиуса звезды [3]. Таким образом, полярные шапки могут рассматриваться в качестве интенсивных источников жесткого излучения пульсаров и магнетаров.

5 Теоретическая и математическая физика, т. 140, № 1, 2004 г. 
Диаграмма направленности рентгеновского и гамма-излучения, генерируемого высокоэнергетичным электроном, движушимся с релятивистской скоростью вдоль магнитной силовой линии, будет довольно узкой и ориентированной по касательной к силовой линии, вдоль которой движется электрон. Поэтому основная часть лучей генерируемого электронами излучения будет лежать в плоскостях магнитных меридианов нейтронной звезды. При уменьшении области излучения все большее число лучей будет попадать в плоскости магнитных меридианов этой звезды. В предельном случае очень малой излучающей области источники излучения можно считать точечными, находящимися строго на двух магнитных полюсах нейтронной звезды. В этом случае, очевидно, все лучи электромагнитных волн будут лежать в плоскостях магнитных меридианов.

Согласно нелинейной электродинамике при распространении электромагнитных волн в сильном магнитном поле нейтронных звезд должны происходить эффекты их нелинейного взаимодействия [6]-[9]. Обсудим возможности для наблюдения нелинейноэлектродинамических эффектов при распространении рентгеновского и гамма-излучения в плоскостях магнитных меридианов пульсаров и магнетаров.

\section{2. ОПРЕДЕЛЕНИЕ МЕТРИЧЕСКОГО ТЕНЗОРА ЭФФЕКТИВНОГО ПРОСТРАНСТВА-ВРЕМЕНИ}

Представим нейтронную звезду с массой $M$, радиусом $R$ и магнитным дипольным моментом m. Рассмотрим законы распространения электромагнитных сигналов в плоскости магнитного меридиана этой звезды, предписываемые нелинейной электродинамикой вакуума.

Так как нейтронные звезды обладают значительным гравитационным полем, то выражение (2) следует записать в общековариантном виде [7]

$$
L=\frac{\sqrt{-g}}{32 \pi}\left\{2 J_{2}+\xi\left[\left(\eta_{1}-2 \eta_{2}\right) J_{2}^{2}+4 \eta_{2} J_{4}\right]\right\}+O\left(\eta \xi^{2} \mathbf{B}^{6}\right)-\frac{\sqrt{-g}}{c} j^{n} A_{n},
$$

где $g$ - определитель метрического тензора исходного псевдориманова пространства-времени, а $J_{2}=F_{i k} F^{k i}$ и $J_{4}=F_{i k} F^{k m} F_{m l} F^{l i}$ - независимые инварианты тензора электромагнитного поля $F_{i k}$.

Гравитационное поле нейтронной звезды будем считать совпадающим с полем Шварцшильда,

$$
g_{00}=1-\frac{r_{\mathrm{g}}}{r}, \quad g_{r r}=-\frac{r}{r-r_{\mathrm{g}}}, \quad g_{\theta \theta}=-r^{2}, \quad g_{\varphi \varphi}=-r^{2} \sin ^{2} \theta
$$

где $r_{\mathrm{g}}$ - гравитационный радиус нейтронной звезды.

Тогда система уравнений нелинейной электродинамики вакуума, получаемая из лагранжиана (3), примет вид

$$
\begin{gathered}
\frac{1}{\sqrt{-g}} \frac{\partial}{\partial x^{n}}\left\{\sqrt{-g} Q^{m n}\right\}=-\frac{4 \pi}{c} j^{m}, \\
\frac{\partial F_{m n}}{\partial x^{k}}+\frac{\partial F_{n k}}{\partial x^{m}}+\frac{\partial F_{k m}}{\partial x^{n}}=0,
\end{gathered}
$$


где для сокрашения записи введено обозначение

$$
Q^{m n}=\left[1+\xi\left(\eta_{1}-2 \eta_{2}\right) J_{2}\right] F^{m n}+4 \xi \eta_{2} F^{m l} F_{l k} F^{k n}
$$

В нулевом приближении по $r_{\mathrm{g}}$ и $\xi$ из уравнений (5) несложно найти магнитное поле звезды, магнитный дипольный момент $\mathbf{m}$ которой лежит в плоскости $X O Y$ и составляет угол $\varphi_{1}$ с положительным направлением оси $x$ :

$$
\begin{aligned}
& F_{13}=-\frac{|\mathbf{m}|}{r^{2}} \sin \theta \cos \theta \cos \left(\varphi-\varphi_{1}\right), \quad F_{32}=\frac{2|\mathbf{m}|}{r} \sin ^{2} \theta \cos \left(\varphi-\varphi_{1}\right), \\
& F_{12}=-\frac{|\mathbf{m}|}{r^{2}} \sin \left(\varphi-\varphi_{1}\right) .
\end{aligned}
$$

Используя систему уравнений (5), найдем уравнение эйконала для слабой высокочастотной электромагнитной волны, распространяющейся в гравитационном (4) и магнитном дипольном (6) полях нейтронной звезды.

В уравнения (5) входят два малых параметра $r_{\mathrm{g}} / r$ и $\xi|\mathbf{m}|^{2} / r^{6}$. Так как для типичных нейтронных звезд вблизи их поверхности $r_{\mathrm{g}} / r \sim 10^{-2}$ и $\xi|\mathbf{m}|^{2} / r^{6} \sim 10^{-4}$, то все вычисления будем проводить с точностью, линейной по $\xi|\mathbf{m}|^{2} / r^{6}$ и квадратичной по $r_{\mathrm{g}} / r$.

Тензор электромагнитного поля, входяший в уравнения (5), представим в виде суммы дипольного магнитного поля $F_{m n}^{(0)}$ звезды $(6)$ и поля слабой электромагнитной волны $f_{m n}$,

$$
F_{m n}=F_{m n}^{(0)}+f_{m n}=F_{m n}^{(0)}+A_{m n}(\mathbf{r}, t) e^{i S(\mathbf{r}, t)}
$$

где, как обычно [10], амплитуда $A_{m n}(\mathbf{r}, t)$ волны является медленно изменяющейся функцией координат и времени, а эйконал $S(\mathbf{r}, t)$ - быстро изменяющейся функцией.

Подставляя выражение (7) в систему уравнений (5), найдем по общим правилам [2], $[6],[11]$ уравнение, которому удовлетворяет эйконал $S$. Для луча, лежашего в плоскости магнитного меридиана $\theta=\pi / 2$ дипольного магнитного поля (6), явный вид этого уравнения из-за эффекта [12] двулучепреломления в магнитном поле зависит от поляризации электромагнитной волны,

$$
\begin{aligned}
\frac{r}{\left(r-r_{\mathrm{g}}\right)} & \left(\frac{\partial S}{\partial x^{0}}\right)^{2}-\left(1-\frac{r_{\mathrm{g}}}{r}\right)\left(\frac{\partial S}{\partial r}\right)^{2}-\frac{1}{r^{2}}\left(\frac{\partial S}{\partial \varphi}\right)^{2}+ \\
+ & \frac{4 \eta \xi \mathbf{m}^{2}}{r^{6}}\left[\sin \left(\varphi-\varphi_{1}\right) \frac{\partial S}{\partial r}-\frac{2 \cos \left(\varphi-\varphi_{1}\right)}{r} \frac{\partial S}{\partial \varphi}\right]^{2}=0
\end{aligned}
$$

где $\eta=\eta_{2}$ для волны, поляризованной в рассматриваемой плоскости магнитного меридиана, и $\eta=\eta_{1}$ для волны, поляризованной перпендикулярно к этой плоскости.

Из уравнения (8) непосредственно следует, что нелинейно-электродинамическое воздействие магнитного поля нейтронной звезды на электромагнитные волны, лучи которых лежат в плоскости магнитного меридиана $\theta=\pi / 2$, эквивалентно введению эффективного псевдориманова пространства-времени, отличные от нуля компоненты метри- 
ческого тензора которого зависят от внешнего магнитного поля и от состояния поляризации слабой электромагнитной волны,

$$
\begin{aligned}
g^{00} & =\frac{r}{r-r_{\mathrm{g}}}, & g^{r r} & =-1+\frac{r_{\mathrm{g}}}{r}+\frac{4 \eta \xi \mathbf{m}^{2}}{r^{6}} \sin ^{2}\left(\varphi-\varphi_{1}\right), \\
g^{r \varphi} & =-\frac{4 \eta \xi \mathbf{m}^{2}}{r^{7}} \sin 2\left(\varphi-\varphi_{1}\right), & g^{\varphi \varphi} & =-\frac{1}{r^{2}}\left[1-\frac{16 \eta \xi \mathbf{m}^{2}}{r^{6}} \cos ^{2}\left(\varphi-\varphi_{1}\right)\right] .
\end{aligned}
$$

Поэтому законы распространения электромагнитных сигналов в плоскости магнитного меридиана нейтронной звезды можно найти, решая уравнение для изотропной геодезической в пространстве-времени с метрическим тензором (9) [6], [11].

\section{3. УРАВНЕНИЯ ДВИЖЕНИЯ ФОТОНОВ В ПЛОСКОСТИ МАГНИТНОГО МЕРИДИАНА НЕЙТРОННОЙ ЗВЕЗДЫ}

Согласно аналитической механике уравнение Гамильтона-Якоби (8) полностью эквивалентно уравнению для изотропной геодезической в пространстве-времени с метрическим тензором (9)

$$
\frac{d K^{i}}{d \sigma}+\Gamma_{n l}^{i} K^{n} K^{l}=0
$$

где $\sigma$ - некоторьй аффинный параметр, $K^{i}=d x^{i} / d \sigma-4$-вектор, касательный к лучу и удовлетворяющий условию $K^{i} K^{n} g_{i n}=0$. В рассматриваемом случае при сделанных предположениях об ориентации вектора дипольного магнитного момента в качестве плоскости меридиана удобно выбрать плоскость $\theta=\pi / 2$. В этой плоскости уравнение для изотропной геодезической (10) запишем в несколько ином виде, переходя к дифференцированию по радиальной координате $r$ в соответствии с равенством

В результате уравнения (10) примут вид

$$
\frac{d}{d \sigma}=k^{r} \frac{d}{d r} \text {. }
$$

$$
\begin{array}{r}
\frac{d^{2} \varphi}{d r^{2}}+\left\{\Gamma_{\alpha \beta}^{\varphi}+\left[\frac{r_{\mathrm{g}}}{2 r^{2}} g_{\alpha \beta}-\Gamma_{\alpha \beta}^{r}\right] \frac{d \varphi}{d r}\right\} \frac{d x^{\alpha}}{d r} \frac{d x^{\beta}}{d r}=0, \\
\frac{d^{2} x^{0}}{d r^{2}}+\frac{d x^{0}}{d r}\left\{2 \Gamma_{0 r}^{0}+\left[\frac{r_{\mathrm{g}}}{2 r^{2}} g_{\alpha \beta}-\Gamma_{\alpha \beta}^{r}\right] \frac{d x^{\alpha}}{d r} \frac{d x^{\beta}}{d r}\right\}=0 .
\end{array}
$$

Подставляя в уравнения (11) соотношения (9), получим

$$
\begin{aligned}
\frac{d^{2} \varphi}{d r^{2}}+ & \frac{2}{r} \frac{d \varphi}{d r}+\left(r-\frac{3 r_{\mathrm{g}}}{2}\right)\left(\frac{d \varphi}{d r}\right)^{3}+\frac{6 \eta_{1,2} \xi \mathbf{m}^{2}}{r^{5}}\left\{\frac{2\left[1-9 \cos ^{2}\left(\varphi-\varphi_{1}\right)\right]}{r^{2}} \frac{d \varphi}{d r}+\right. \\
& \left.+\frac{3 \sin 2\left(\varphi-\varphi_{1}\right)}{r^{3}}-\frac{4 \sin 2\left(\varphi-\varphi_{1}\right)}{r}\left(\frac{d \varphi}{d r}\right)^{2}-2\left[1+\cos ^{2}\left(\varphi-\varphi_{1}\right)\right]\left(\frac{d \varphi}{d r}\right)^{3}\right\}=0, \\
\frac{d^{2} x^{0}}{d r^{2}}+ & \left\{\left(r-\frac{3 r_{\mathrm{g}}}{2}\right)\left(\frac{d \varphi}{d r}\right)^{2}+\frac{r_{\mathrm{g}}}{r^{2}}\left(1+\frac{r_{\mathrm{g}}}{r}\right)+\frac{12 \eta_{1,2} \xi \mathbf{m}^{2}}{r^{5}}\left[\frac{\sin ^{2}\left(\varphi-\varphi_{1}\right)}{r^{2}}-\right.\right. \\
& \left.\left.-\frac{\sin 2\left(\varphi-\varphi_{1}\right)}{r} \frac{d \varphi}{d r}-\left[1+\cos ^{2}\left(\varphi-\varphi_{1}\right)\right]\left(\frac{d \varphi}{d r}\right)^{2}\right]\right\} \frac{d x^{0}}{d r}=0 .
\end{aligned}
$$


Проинтегрируем первое уравнение этой системы методом последовательных приближений с принятой точностью:

$$
\begin{aligned}
\varphi= & \varphi_{0}+\arcsin \frac{\alpha}{r}+r_{\mathrm{g}}\left\{C_{0}+\frac{C_{1}}{\sqrt{r^{2}-\alpha^{2}}}-\frac{r}{\alpha \sqrt{r^{2}-\alpha^{2}}}+\frac{\alpha}{2 r \sqrt{r^{2}-\alpha^{2}}}\right\}+ \\
& +r_{\mathrm{g}}^{2}\left\{C_{2}+\frac{C_{3}}{\sqrt{r^{2}-\alpha^{2}}}+\frac{15}{16 \alpha^{2}} \arcsin \frac{\alpha}{r}+\frac{1}{\left(\sqrt{r^{2}-\alpha^{2}}\right)^{3}}\left[\frac{C_{1} r\left(2 r^{2}-3 \alpha^{2}\right)}{2 \alpha^{2}}+\right.\right. \\
& \left.\left.+\frac{C_{1}^{2}\left(3 r^{2}-2 \alpha^{2}\right)}{2 \alpha}-\frac{15 r^{2}}{16 \alpha}+\frac{5 \alpha}{4}-\frac{3 \alpha^{3}}{16 r^{2}}\right]\right\}+\eta_{1,2} \xi \mathbf{m}^{2}\left\{C_{4}+\frac{C_{5}}{\sqrt{r^{2}-\alpha^{2}}}+\right. \\
& +\frac{3}{64 \alpha^{6}}\left[140-65 \cos 2\left(\varphi_{0}-\varphi_{1}\right)+\frac{26 \alpha}{\sqrt{r^{2}-\alpha^{2}}} \sin 2\left(\varphi_{0}-\varphi_{1}\right)\right] \arcsin \frac{\alpha}{r}- \\
& -\frac{\sin 2\left(\varphi_{0}-\varphi_{1}\right)}{32 r^{8} \alpha^{4}}\left[39 r^{6}+26 r^{4} \alpha^{2}+40 r^{2} \alpha^{4}-72 \alpha^{6}\right]+ \\
& +\frac{\cos 2\left(\varphi_{0}-\varphi_{1}\right)}{64 r^{8} \alpha^{5} \sqrt{r^{2}-\alpha^{2}}}\left[195 r^{8}-65 r^{6} \alpha^{2}-26 r^{4} \alpha^{4}-152 r^{2} \alpha^{6}+144 \alpha^{8}\right]- \\
& \left.-\frac{\left[105 r^{6}-35 r^{4} \alpha^{2}-14 r^{2} \alpha^{4}+8 \alpha^{6}\right]}{16 r^{6} \alpha^{5} \sqrt{r^{2}-\alpha^{2}}}\right\},
\end{aligned}
$$

где $\alpha, \varphi_{0}, C_{0}, C_{1}, C_{2}, C_{3}, C_{4}$ и $C_{5}$ - постоянные интегрирования соответствуюших приближений.

Часть этих постоянных можно определить, потребовав, чтобы луч начинался в источнике жесткого излучения, который находится на магнитном полюсе нейтронной звезды в точке $r=R, \varphi=\varphi_{1}$. Тогда, вводя вспомогательный угол $\beta(-\pi / 2<\beta<\pi / 2)$ в соответствии с соотношением $\alpha=R \sin \beta$, будем иметь

$$
\begin{aligned}
\varphi_{0}= & \varphi_{1}-\beta, \quad C_{0}=-\frac{C_{1}}{R \cos \beta}+\frac{1}{R \sin \beta \cos \beta}-\frac{\sin \beta}{2 R \cos \beta}, \\
C_{2}= & -\frac{C_{3}}{R \cos \beta}-\frac{15 \beta}{16 R^{2} \sin ^{2} \beta}-\frac{C_{1}\left(2-3 \sin ^{2} \beta\right)}{2 R^{2} \sin ^{2} \beta \cos ^{3} \beta}-\frac{C_{1}^{2}\left(3-2 \sin ^{2} \beta\right)}{2 R^{2} \sin \beta \cos ^{3} \beta}+ \\
& +\frac{15}{16 R^{2} \sin \beta \cos ^{3} \beta}-\frac{5 \sin \beta}{4 R^{2} \cos ^{3} \beta}+\frac{3 \sin ^{3} \beta}{16 R^{2} \cos ^{3} \beta}, \\
C_{4}= & -\frac{C_{5}}{R \cos \beta}+\frac{1}{64 R^{6} \sin ^{6} \beta \cos \beta}\left[76 \sin ^{7} \beta-108 \sin ^{5} \beta+\right. \\
& \left.+159 \sin ^{3} \beta+225 \sin \beta-\beta \cos \beta\left(225+234 \sin ^{2} \beta\right)\right] .
\end{aligned}
$$

Для определения оставшихся постоянных интегрирования выберем из семейства лучей, выходящих из точки $r=R, \varphi=\varphi_{1}$, луч, который проходит через точку $r=r_{2}, \varphi=\varphi_{2}$, где помешен детектор рентгеновского и гамма-излучения.

Так как пульсары и магнетары находятся на значительных расстояниях от Земли 
$\left(r_{2}>1\right.$ кпк $)$, то в дальнейших расчетах будем полагать $r_{2} \rightarrow \infty$. В результате получим

$$
\begin{aligned}
\beta= & \varphi_{1}-\varphi_{2}, \quad \varphi_{0}=\varphi_{2}, \\
C_{0}= & \frac{1}{R \sin \beta}, \quad C_{1}=\frac{(1-\cos \beta)}{\sin \beta}-\frac{\sin \beta}{2}, \quad C_{4}=0, \\
C_{2}= & \frac{2(\cos \beta-1)+\sin ^{2} \beta}{2 R^{2} \sin ^{3} \beta}, \\
C_{3}= & \frac{1}{16 R \sin ^{3} \beta}\left[79 \sin ^{2} \beta+80 \cos \beta-7 \sin ^{4} \beta-\right. \\
& \left.-24 \sin ^{2} \beta \cos \beta-15 \beta \sin \beta \cos \beta-80\right], \\
C_{5}= & \frac{1}{64 R^{5} \sin ^{6} \beta}\left[76 \sin ^{7} \beta-108 \sin ^{5} \beta+\right. \\
& \left.+159 \sin ^{3} \beta+225 \sin \beta-\left(225+234 \sin ^{2} \beta\right) \beta \cos \beta\right] .
\end{aligned}
$$

Таким образом, луч, выходяший из точки $r=R, \varphi=\varphi_{1}$ и проходяший через детектор рентгеновского и гамма-излучения, находящийся в точке $r=r_{2} \gg R, \varphi=\varphi_{2}$, описывается уравнением (13), если в нем учесть соотношения (14).

Проинтегрируем теперь второе уравнение системы (12):

$$
\begin{aligned}
x^{0}= & c t_{0}+\sqrt{r^{2}-\alpha^{2}}+r_{\mathrm{g}}\left[Q_{0}+Q_{1} \sqrt{r^{2}-\alpha^{2}}-\frac{C_{1}\left(2 \alpha^{2}-r^{2}\right)}{\alpha \sqrt{r^{2}-\alpha^{2}}}+\right. \\
& \left.+\frac{r}{2 \sqrt{r^{2}-\alpha^{2}}}+\ln \left(r+\sqrt{r^{2}-\alpha^{2}}\right)\right]+ \\
& +r_{\mathrm{g}}^{2}\left[Q_{2}+Q_{3} \sqrt{r^{2}-\alpha^{2}}+\left(Q_{1}+\frac{C_{1}}{\alpha}\right) \ln \left(r+\sqrt{r^{2}-\alpha^{2}}\right)-\right. \\
& -\frac{15}{8 \alpha} \arcsin \frac{\alpha}{r}+\frac{\left(C_{1} Q_{1}+C_{3}\right)\left(r^{2}-3 \alpha^{2}\right)}{2 \alpha \sqrt{r^{2}-\alpha^{2}}}+\frac{C_{1}^{2}\left(3 r^{4}-12 \alpha^{2} r^{2}+8 \alpha^{4}\right)}{2 \alpha^{2}\left(\sqrt{r^{2}-\alpha^{2}}\right)^{3}}+ \\
& \left.+\frac{\left(4 r^{4}-\alpha^{2} r^{2}-4 \alpha^{4}\right)}{8 \alpha^{2}\left(\sqrt{r^{2}-\alpha^{2}}\right)^{3}}+\frac{Q_{1} r}{2 \sqrt{r^{2}-\alpha^{2}}}+\frac{C_{1} r\left(2 \alpha^{2}-r^{2}\right)}{2 \alpha\left(\sqrt{r^{2}-\alpha^{2}}\right)^{3}}\right]+ \\
& +\eta_{1,2} \xi \mathbf{m}^{2}\left\{Q_{4}+Q_{5} \sqrt{r^{2}-\alpha^{2}}-\frac{3}{32 \alpha^{5}}\left[84-39 \cos 2\left(\varphi_{2}-\varphi_{1}\right)+\right.\right. \\
& \left.+\frac{13 \alpha}{\sqrt{r^{2}-\alpha^{2}}} \sin 2\left(\varphi_{2}-\varphi_{1}\right)\right] \arcsin \frac{\alpha}{r}+\frac{\sin 2\left(\varphi_{2}-\varphi_{1}\right)}{32 r^{6} \alpha^{3}}\left[39 r^{4}+26 r^{2} \alpha^{2}+8 \alpha^{4}\right]- \\
& -\frac{\cos 2\left(\varphi_{2}-\varphi_{1}\right)}{32 r^{6} \alpha^{4} \sqrt{r^{2}-\alpha^{2}}}\left[117 r^{6}-39 r^{4} \alpha^{2}-22 r^{2} \alpha^{4}-8 \alpha^{6}\right]+\frac{C_{5}\left(r^{2}-2 \alpha^{2}\right)}{\alpha \sqrt{r^{2}-\alpha^{2}}}+ \\
& \left.+\frac{\left[63 r^{4}-21 r^{2} \alpha^{2}-10 \alpha^{4}\right]}{8 r^{4} \alpha^{4} \sqrt{r^{2}-\alpha^{2}}}\right\}
\end{aligned}
$$

где $t_{0}, Q_{0}, Q_{1}, Q_{2}, Q_{3}, Q_{4}$ и $Q_{5}$ - постоянные интегрирования.

Следует отметить, что время $t$, входящее в выражение (15), измеряется по часам наблюдателя, находящегося вдали $(r \gg R)$ от нейтронной звезды. 
Для определения постоянных интегрирования $t_{0}, Q_{0}, Q_{2}$ и $Q_{4}$ предположим, что электромагнитный сигнал излучается из точки $r=R, \varphi=\varphi_{1}$ в момент времени $t=0$ по часам удаленного наблюдателя. Это условие даст только часть необходимых уравнений.

Продифференцируем теперь выражения (13) и (15) по $r$. Подставим полученные выражения в соотношение $g_{i k} d x^{i} d x^{k}=0$, которому должен удовлетворять процесс распространения электромагнитных волн в произвольном псевдоримановом пространстве-времени. В результате найдем недостающее уравнение для определения постоянных интегрирования. Учитывая соотношения (14) и разрешая полученные уравнения методом последовательных приближений, получим

$$
\begin{aligned}
t_{0}= & -\frac{R}{c} \cos \beta, \quad Q_{0}=\frac{\cos \beta}{2}-1-\ln [R(1+\cos \beta)], \\
Q_{1}= & \frac{1}{R}\left[\frac{1}{2}-\frac{1-\cos \beta}{\sin ^{2} \beta}\right], \quad Q_{2}=\frac{1}{16 R}\left[\cos \beta+\frac{15 \beta}{\sin \beta}\right], \\
Q_{3}= & \frac{1}{32 R^{2} \sin ^{4} \beta}\left[16(1-\cos \beta)+15 \beta \sin \beta \cos \beta-\left(31+8 \cos \beta+\sin ^{2} \beta\right) \sin ^{2} \beta\right], \\
Q_{4}= & \frac{\left[\left(45+78 \sin ^{2} \beta\right) \beta-\left(45+108 \sin ^{2} \beta+76 \sin ^{4} \beta\right) \sin \beta \cos \beta\right]}{64 R^{5} \sin ^{5} \beta}, \\
Q_{5}= & \frac{1}{64 R^{6} \sin ^{7} \beta}\left[\left(225+234 \sin ^{2} \beta\right) \beta \cos \beta-\right. \\
& \left.-76 \sin ^{7} \beta+108 \sin ^{5} \beta-159 \sin ^{3} \beta-225 \sin \beta\right] .
\end{aligned}
$$

Соотношения (13)-(16) описывают в параметрическом виде лучи, по которым фотоны распространяются в плоскости магнитного меридиана из источника, находящегося на одном из магнитных полюсов нейтронной звезды, к удаленному детектору. Они позволяют проанализировать нелинейно-электродинамическое и гравитационное искривления лучей в плоскости магнитного меридиана нейтронной звезды и исследовать законы распространения электромагнитных сигналов с различной поляризацией по этим лучам.

Следует, однако, отметить [11], [13], что хотя угол искривления может достигать весьма больших значений, $\delta \varphi \sim 10^{-4}$ рад $\sim 1^{\prime}$, измерить его с Земли невозможно из-за большой удаленности пульсаров и магнетаров (более десятка кпк).

\section{4. ВЫЧИСЛЕНИЕ ВРЕМЕНИ НЕЛИНЕЙНО-ЭЛЕКТРОДИНАМИЧЕСКОГО ЗАПАЗДЫВАНИЯ ЭЛЕКТРОМАГНИТНЫХ СИГНАЛОВ}

Рассмотрим два электромагнитных сигнала, излучаемых в один и тот же момент времени из точки $r=R, \varphi=\varphi_{1}$ и принимаемых детектором, расположенным в точке $r=r_{2}, \varphi=\varphi_{2}$. Предположим, что первый сигнал поляризован в плоскости магнитного меридиана, а второй - перпендикулярно к этой плоскости.

Так как эти сигналы распространяются по разным лучам и с разной скоростью, то в точку наблюдения они придут неодновременно. Вычислим время нелинейно-электродинамического запаздывания первого сигнала по сравнению со вторым. 
Время, необходимое для распространения первого сигнала от источника к наблюдателю, в силу соотношений (15) и (16) будет равно

$$
\begin{aligned}
x^{0}\left(r_{2}\right)= & c t_{1}=r_{2}-R \cos \beta+\frac{r_{\mathrm{g}}}{2}\left[\cos \beta-1+2 \ln \frac{2 r_{2}}{R(1+\cos \beta)}\right]+ \\
& +\frac{r_{\mathrm{g}}^{2}\left[16(\cos \beta-1)+15 \beta \sin \beta+(8+\cos \beta) \sin ^{2} \beta\right]}{16 R \sin ^{2} \beta}+ \\
& +\frac{\eta_{1} \xi \mathbf{m}^{2}}{64 R^{5} \sin ^{5} \beta}\left\{\left(45+78 \sin ^{2} \beta\right) \beta-\left(45+108 \sin ^{2} \beta+76 \sin ^{4} \beta\right) \sin \beta \cos \beta\right\} .
\end{aligned}
$$

Для второго сигнала имеем аналогичное выражение, получаемое из равенства (17) заменой $\eta_{1}$ на $\eta_{2}$.

Поэтому время запаздывания первого сигнала по отношению ко второму

$$
\Delta t=t_{1}-t_{2}=\frac{\left(\eta_{1}-\eta_{2}\right) \xi \mathbf{m}^{2}}{64 c R^{5} \sin ^{5} \beta}\left\{\left(45+78 \sin ^{2} \beta\right) \beta-\left(45+108 \sin ^{2} \beta+76 \sin ^{4} \beta\right) \sin \beta \cos \beta\right\} .
$$

Проанализируем полученное выражение. При изменении угла $\beta$ от $\pi / 2$ (выход изображения источника из-за горизонта нейтронной звезды) до нуля (радиальное распространение электромагнитного сигнала с магнитного полюса к наблюдателю) и далее до $-\pi / 2$ (уход изображения источника за горизонт нейтронной звезды с другой стороны ее диска) величина $\Delta t$ изменяется от $123 \pi\left(\eta_{1}-\eta_{2}\right) \xi \mathbf{m}^{2} /\left(128 c R^{5}\right)$ до нуля, а затем снова от нуля до $123 \pi\left(\eta_{1}-\eta_{2}\right) \xi \mathbf{m}^{2} /\left(128 c R^{5}\right)$. Отсюда следует, что при $\eta_{1}>\eta_{2}$ электромагнитный сигнал, поляризованный перпендикулярно к плоскости магнитного меридиана, придет к детектору раньше (быстрая мода), чем электромагнитный сигнал, поляризованный в плоскости магнитного меридиана (медленная мода). Если же $\eta_{1}<\eta_{2}$, то все должно происходить наоборот.

Величина промежутка времени $\Delta t$ сушественно зависит от разности $\eta_{1}-\eta_{2}$ постмаксвелловских параметров, и поэтому она различна в различных моделях нелинейной электродинамики вакуума. В частности, в нелинейной электродинамике Гейзенберга-Эйлера эта величина для типичных пульсаров может достигать значения 1 мкс, в то время как в теории Борна-Инфельда она строго равна нулю.

С экспериментальной точки зрения эффект запаздывания электромагнитных сигналов, излучаемых с магнитных полюсов неврашаюшейся нейтронной звезды, будет проявляться по-разному в зависимости от того, непрерьвное или импульсное излучение она испускает. Будем считать, что это излучение имеет хаотическую поляризацию.

В случае непрерывного излучения временна́я зависимость принимаемого излучения на одной поляризации будет сдвинута относительно временно́й зависимости интенсивности рентгеновского и гамма-излучения на ортогональной ей поляризации, причем величина временно́го сдвига будет зависеть от угла $\beta$ меж ду вектором магнитного момента звезды и радиус-вектором точки, в которой находится детектор.

В случае излучения отдельных рентгеновских и гамма-импульсов (пакетов фотонов), длительность которых больше времени $\Delta t(18)$, эффект запаздывания будет проявляться по-другому. При излучении произвольно поляризованных импульсов принимаемые 
импульсы будут иметь переменную поляризацию по их длине: при $\eta_{1}>\eta_{2}$ передняя часть импульса длительностью $\tau=\Delta t$ должна быть поляризована перпендикулярно к плоскости магнитного меридиана нейтронной звезды, а остальная часть импульса в обшем случае будет поляризована хаотично.

Предположим теперь, что рассматриваемая нами нейтронная звезда врашается с частотой $\Omega$ вокруг оси, перпендикулярной вектору магнитного дипольного момента $\mathbf{m}$ (так называемый случай “перпендикулярного ротатора"). Согласно работам [14], [15] и в этом случае сохраняются условия для истечения зарядов с поверхности нейтронной звезды и заполнения ее магнитосферы. Поэтому обеспечивается необходимое условие для генерации жесткого излучения - вблизи нейтронной звезды появляются свободные электроны, которые могут ускоряться в продольном электрическом поле.

Будем считать, что угловая скорость $\Omega$ врашения этой звезды достаточно мала, в результате чего линейная скорость точек на поверхности звезды значительно меньше скорости света. В этом случае время распространения электромагнитных сигналов в области $r \sim 5 R$ сильного магнитного поля звезды, где осушествляется главная часть нелинейно-электродинамического и гравитационного воздействий на эти сигналы, будет значительно меньше периода врашения звезды. Тогда область взаимодействия оказывается в ближней зоне магнитного дипольного излучения и все полученные нами формулы сохраняют свою силу. Подставив в выражение (18) $\beta=\Omega t$, получим

$\Delta t=\frac{\left(\eta_{1}-\eta_{2}\right) \xi \mathbf{m}^{2}}{512 c R^{5} \sin ^{5} \Omega t}\{[672-312 \cos (2 \Omega t)] \Omega t-19 \sin (6 \Omega t)+184 \sin (4 \Omega t)-491 \sin (2 \Omega t)\}$.

Отсюда следует, что время запаздывания сигналов с двумя главными поляризациями будет модулировано из-за врашения звезды.

В частности, в случае непрерывного излучения временна́я зависимость принимаемого излучения на одной поляризации будет совершать колебания относительно временно́й зависимости интенсивности рентгеновского и гамма-излучения на ортогональной ей поляризации, причем временной сдвиг будет изменяться по закону (19).

Как известно, реальные временны́е профили рентгеновского и гамма-излучения пульсаров выглядят как периодическая последовательность импульсов (пульсаций), период которой равен периоду вращения нейтронной звезды, а длительность пульсации определяется в первую очередь шириной диаграммы направленности излучения. В случае очень узкой диаграммы направленности временной профиль излучения пульсара может быть представлен как периодическая последовательность импульсов, длительность которых много меньше периода следования. При $\eta_{1}>\eta_{2}$, очевидно, передний фронт импульсов должен характеризоваться линейной поляризацией, плоскость которой перпендикулярна к плоскости магнитного меридиана нейтронной звезды. При этом, как следует из формулы (19), длительность этой поляризованной передней части импульса будет изменяться от нуля до максимального значения по закону (19).

Поскольку длительность импульса $\tau$ меньше периода $2 \pi / \Omega$, то за время $\tau$ это периодическое изменение будет несушественным. Поэтому при анализе реальных сигналов можно считать длительность поляризованной части импульса постоянной, величина 
которой будет зависеть от ориентации линии наблюдений относительно плоскости меридиана нейтронной звезды. Для того чтобы экспериментально зарегистрировать такое изменение, потребуются детальные поляризационные измерения, чтобы построить среднюю кривую пульсаций для линейно-поляризованной и неполяризованной компонент импульса с высоким временным разрешением ( 1 мкс). Следует отметить, что современная техника регистрации рентгеновских и гамма-фотонов позволяет определять время задержки их прихода с точностью $\leqslant 0.1$ мкс, что сушественно меньше времени ожидаемой задержки фотонов с разной “нелинейно-электродинамической” поляризацией.

\section{5. ЗАКЛЮЧЕНИЕ}

Таким образом, в результате нелинейно-электродинамического и гравитационного воздействий лучи электромагнитных волн, излучаемых с магнитных полюсов нейтронных звезд, искривляются и при $\eta_{1} \neq \eta_{2}$ время распространения сигналов с поверхности звезды к наблюдателю для двух нормальных мод различно. К сожалению, из-за чрезвычайной удаленности пульсаров и магнетаров от Земли (более десятка кпк), наблюдать эффект нелинейно-электродинамического искривления лучей невозможно. Однако современный уровень развития экспериментальной техники позволяет, в принципе, наблюдать проявления эффекта запаздывания сигналов, имеюших различную поляризацию.

Благодарности. Работа выполнена при поддержке РФФИ, грант № 02-02-16598.

\section{Список литературы}

[1] D. L. Burke, R. C. Feld, G. Horton-Smith et al. Phys. Rev. Lett. 1997. V. 79. P. 1626.

[2] В. И. Денисов, И. П. Денисова. Докл. РАН. 2001. Т. 378. № 4. С. 463.

[3] A. K. Harding, A. Muslimov. Astrophys. J. 1998. V. 508. P. 328.

[4] P. A. Sturrock. Astrophys. J. 1971. V. 164. P. 529.

[5] K. S. Cheng, C. Ho, M. A. Ruderman. Astrophys. J. 1986. V. 300. P. 500.

[6] В. И. Денисов. ТМФ. 2002. Т. 132. № 2. С. 211.

[7] П. А. Виивцева, В. И. Денисов, И. П. Денисова. Докл. РАН. 2002. Т. 387. № 2. С. 178.

[8] В. И. Денисов, И. П. Денисова, И. В. Кривченков. ЖЭТФ. 2002. Т. 122. № 8. С. 227.

[9] В. И. Денисов, И. П. Денисова, С. И. Свертилов. ТМФ. 2003. Т. 135. № 2. С. 322.

[10] Л. Д. Ландау, Е. М. Лифииц. Теория поля. М.: Наука, 1988.

[11] В. И. Денисов, И. П. Денисова, И. В. Кривченков. Докл. РАН. 2003. Т. 388. № 1. С. 23.

[12] И. М. Тернов, В. Р. Халилов, В. Н. Родионов. Взаимодействие заряженных частиц с сильным электромагнитным полем. М.: МГУ, 1982.

[13] V. I. Denisov, S. I. Svertilov. Astron. Astrophys. 2003. V. 399. P. L39.

[14] L. M. Cohen, E. T. Toton. Astrophys. Lett. 1971. V. 7. P. 213.

[15] L. Mestel. Nature Phys. Sci. 1971. V. 233. P. 149.

Поступила в редакцию 17.VII.2003 г. 